\title{
Functional Analysis of the Mouse Scn8a Sodium Channel
}

\author{
Marianne R. Smith, ${ }^{1}$ Raymond D. Smith, ${ }^{1}$ Nicholas W. Plummer, ${ }^{2}$ Miriam H. Meisler, ${ }^{2}$ and Alan L. Goldin ${ }^{1}$ \\ ${ }^{1}$ Department of Microbiology and Molecular Genetics and Physiology and Biophysics, University of California, Irvine, \\ California 92697-4025, and 2Department of Human Genetics, University of Michigan, Ann Arbor, Michigan 48109-0618
}

The mouse Scn8a sodium channel and its ortholog Na6 in the rat are abundantly expressed in the CNS. Mutations in mouse Scn8a result in neurological disorders, including paralysis, ataxia, and dystonia. In addition, Scn8a has been observed to mediate unique persistent and resurgent currents in cerebellar Purkinje cells (Raman et al., 1997). To examine the functional characteristics of this channel, we constructed a full-length cDNA clone encoding the mouse Scn8a sodium channel and expressed it in Xenopus oocytes. The electrophysiological properties of the Scn8a channels were compared with those of the Rat1 and Rat2 sodium channels. Scn8a channels were sensitive to tetrodotoxin at a level comparable to that of Rat1 or Rat2. Scn8a channels inactivated more rapidly and showed differences in their voltage-dependent properties compared with Rat1 and Rat2 when only the $\alpha$ subunits were expressed.

Voltage-gated sodium channels play a critical role in the generation of action potentials in excitable cells throughout the nervous system (Catterall, 1992). Many sodium channel isoforms have been identified in the CNS, including Rat1 (Noda et al., 1986), Rat2 (Noda et al., 1986), a splice variant termed Rat2A (Auld et al., 1988), Rat3 (Kayano et al., 1988; Joho et al., 1990), Rat6 (Schaller et al., 1995), and the Rat6 mouse ortholog Scn8a (Burgess et al., 1995). Scn8a/Rat6 is abundantly expressed in neurons throughout the brain and spinal cord (Schaller et al., 1995) and is the major contributor to sodium current in postnatal motor neurons (Garcia et al., 1998). Rat1 is expressed predominantly in the caudal regions and spinal cord, whereas Rat2 is found most abundantly in the rostral regions of the CNS (Gordon et al., 1987; Beckh et al., 1989). In the cerebellum, both Rat1 and Rat6 are found at high levels in the Purkinje cells, whereas Rat2 is expressed in granule cells and Purkinje cells (Furuyama et al., 1993; Black et al., 1994; Schaller et al., 1995; Felts et al., 1997; VegaSaenz de Miera et al., 1997). The $\beta_{1}$ and $\beta_{2}$ subunits are also present in the majority of these cells and can modulate the

Received March 3, 1998; revised May 22, 1998; accepted May 27, 1998.

This work was supported by National Institutes of Health Grants NS26729 (A.L.G.) and NS34609 (M.H.M.). N.W.P. was supported by the Organogenesis Center, University of Michigan. A.L.G. is an Established Investigator of the American Heart Association. We thank Drs. Michael Pugsley, Ted Shih, and Daniel Allen for helpf ul discussions during the course of this work, Dr. Leslie Sprunger for critical review of this manuscript, and Mimi Reyes for excellent technical assistance.

Correspondence should be addressed to Alan L. Goldin, Department of Microbiology and Molecular Genetics and Physiology and Biophysics, University of California, Irvine, CA 92697-4025.

Dr. R. Smith's present address: Department of Biology, University of California, San Diego, La Jolla, CA 92093-0357.

Dr. Plummer's present address: Department of Genetics, Duke University Medical Center, Durham, NC 27710.

Copyright (C) 1998 Society for Neuroscience $\quad 0270-6474 / 98 / 186093-10 \$ 05.00 / 0$
Coexpression of the $\beta_{1}$ and $\beta_{2}$ subunits modulated the properties of Scn8a channels, but to a lesser extent than for the Rat1 or Rat2 channels. Therefore, all three channels showed similar voltage dependence and inactivation kinetics in the presence of the $\beta$ subunits. Scn8a channels coexpressed with the $\beta$ subunits exhibited a persistent current that became larger with increasing depolarization, which was not observed for either Rat1 or Rat2 channels. The unique persistent current observed for Scn8a channels is consistent with the hypothesis that this channel is responsible for distinct sodium conductances underlying repetitive firing of action potentials in Purkinje neurons.

Key words: sodium channel; cloning; expression; Xenopus oocytes; brain; RT-PCR; Purkinje cells; resurgent current; persistent current electrophysiological properties of the channels (Isom et al., 1992, 1995; Smith and Goldin, 1998).

The Scn8a/Rat6 and Rat1 channels may be responsible for distinct sodium conductances in Purkinje cells. Vega-Saenz de Miera et al. (1997) suggested that inactivating and persistent sodium conductances in Purkinje cells result from expression of Rat1 and Rat6, respectively. Raman et al. (1997) compared normal Purkinje cells with those from Scn8a null mice. In Scn8a null cells, there was a reduction in persistent, noninactivating current compared with transient current, suggesting that Scn8a may be predominantly responsible for the persistent current. They also identified a resurgent current that was elicited by an action potential-like waveform in normal Purkinje cells, and this current was significantly decreased in cells from Scn8a null mice.

Several mutations in Scn8a have been characterized, resulting in mice with a variety of symptoms ranging from mild ataxia to dystonia, paralysis, and juvenile lethality (Meisler et al., 1997). These mutations include med and $m e d^{\text {tg }}$, both of which result in complete disruption of the Scn8a gene (Burgess et al., 1995; Kohrman et al., 1995, 1996b). Another mutation, med $^{j o}$, is a single point mutation of Ala to Thr in the domain III S4-S5 linker (Kohrman et al., 1996a). This mutation produces an ataxic phenotype, which may be caused by changes in the voltage-dependent properties of the Scn8a channel (Kohrman et al., 1996a). Both the null and mis-sense mutation have been shown to disrupt spontaneous and repetitive firing of Purkinje cells (Dick et al., 1985; Harris et al., 1992; Raman et al., 1997), suggesting that Scn8a plays a critical role in the firing properties of Purkinje neurons.

Given the fundamental importance of the Scn8a channel, we constructed a full-length cDNA clone encoding Scn8a to characterize its functional properties in Xenopus oocytes. Most of the electrophysiological properties of Scn8a sodium channels were 
similar to those of Rat1 and Rat2 channels and were modulated by the accessory $\beta$ subunits. However, Scn 8 a sodium channels demonstrated a persistent current that was distinctly different from Rat1 or Rat 2 currents.

\section{MATERIALS AND METHODS}

Reverse transcription and PCR. Total RNA was isolated from C57BL/6J adult mice brains using the Trizol reagent (Life Technologies, Gaithersburg, MD). RNA was suspended in sterile, RNase-free water at a concentration of $1.36 \mathrm{mg} / \mathrm{ml}$ and stored at $-75^{\circ} \mathrm{C}$. Mouse brain RNA was reverse-transcribed using an oligo- $\mathrm{dT}_{18}$ primer. The $\mathrm{Scn} 8$ a coding region was amplified by PCR using primers that hybridize to the $5^{\prime}$ and $3^{\prime}$ ends of the published sequence (Burgess et al., 1995). For the upstream primer, a T7 RNA polymerase recognition sequence was incorporated 5' to the Scn8a sequence so that RNA could be transcribed by T7 RNA polymerase directly from the PCR product in vitro. The two PCR primers were 5' end: 5'-GCGCGC(GAATTC)[TAATACGACTCACTATA]GAGAAGATGGCAGCGCGGG-3'; and 3' end: 5'-GCG(GACGTC)TCTGTGTCCGTGAGATTCGG- ${ }^{\prime}$. The $5^{\prime}$ primer contains a six-base G-C clamp, an EcoRI restriction site (in parentheses), a T7 promoter (in brackets), and sense Scn8a DNA sequence consisting of five bases upstream of the Scn8a coding region and 13 bases of coding sequence beginning with the translational start codon (underlined). The $3^{\prime}$ primer contains a threebase G-C clamp, an AatII restriction site (in parentheses), and antisense DNA sequence 67 bases downstream of the $S \operatorname{sen} 8$ a stop codon.

Total brain RNA $(2.8 \mu \mathrm{g})$ was heat-denatured at $65^{\circ} \mathrm{C}$ for $5 \mathrm{~min}$, followed by rapid cooling on ice. Reverse transcription was performed with $0.5 \mathrm{~mm}$ deoxynucleotide triphosphates, $10 \mathrm{~mm}$ dithiothreitol, 100 pmol of oligonucleotide primers, $40 \mathrm{U}$ of RNasin (Promega, Madison, WI), and $500 \mathrm{U}$ of Moloney murine leukemia virus reverse transcriptase (Life Technologies) in a total volume of $50 \mu$ l. Reactions were incubated at room temperature for $5 \mathrm{~min}$ and then at $37^{\circ} \mathrm{C}$ for $2 \mathrm{hr}$. Reaction products were purified by phenol and chloroform extraction and ethanol precipitation and were resuspended in $10 \mu \mathrm{l}$ of distilled water.

The RT product was amplified using the PCR primers described above. One microliter $(1 / 10)$ of the RT product was combined with $1 \mathrm{mM} \mathrm{MgCl}_{2}$, $200 \mu \mathrm{mol}$ of deoxynucleotide triphosphates, a $0.2 \mu \mathrm{M}$ concentration of each primer, and 2.5 U of LA Taq Polymerase (PanVera, Madison, WI). Thermal cycle parameters were one cycle consisting of denaturation at $94^{\circ} \mathrm{C}$ for $4 \mathrm{~min}$, annealing at $53^{\circ} \mathrm{C}$ for $3 \mathrm{~min}$, and polymerase extension at $72^{\circ} \mathrm{C}$ for $6 \mathrm{~min}$, followed by 30 cycles consisting of denaturation at $95^{\circ} \mathrm{C}$ for $30 \mathrm{sec}$, annealing at $53^{\circ} \mathrm{C}$ for $1 \mathrm{~min}$, and polymerase extension at $72^{\circ} \mathrm{C}$ for $6 \mathrm{~min}$. A single PCR fragment of $\sim 6 \mathrm{~kb}$ in size was extracted with phenol and chloroform, precipitated with ethanol, and resuspended in RNase-free water.

Cloning of Scn8a. To ensure that the PCR product encoded a functional sodium channel, RNA was transcribed directly from a sample of the product using a T7 Message Machine RNA transcription kit (Ambion, Austin, TX). Injection of RNA into Xenopus oocytes resulted in expression of up to $2 \mu \mathrm{A}$ of whole-cell tetrodotoxin (TTX)-sensitive inward sodium current, as measured with a two-electrode voltage clamp.

A full-length plasmid containing the Scn8a sequence was constructed by cutting the functionally confirmed PCR product with EcoRI and AatII, and ligating the fragment into pLCT1-A, a modified version of pLCT1 (Smith and Goldin, 1998). pLCT1 was modified by inserting unique EcoRI and AatII sites into the existing unique BglII site. The resulting plasmid, pNaScn8a, was linearized with NotI and transcribed using a T7 Message Machine transcription kit. Injection of undiluted RNA into oocytes resulted in currents as large as $100 \mu \mathrm{A}$ after 48 of incubation at $20^{\circ} \mathrm{C}$. The DNA sequence of pNaScn 8 a was determined at the University of Michigan DNA Sequencing Core Facility (R. Lyons, Director) using the dideoxy chain termination method and the Taq dye terminator cycle sequencing kit with Applied Biosystems (Foster City, CA) 373 Stretch and 377 sequencers.

Expression and electrophysiology. RNA transcripts were synthesized from NotI-linearized DNA templates using a T7 RNA polymerase Message Machine transcription kit (Ambion). The yield of RNA was estimated by glyoxal gel analysis, and pNaScn8a RNA was diluted 1:2000 to result in currents in the range of $2-5 \mu \mathrm{A}$. Stage 5 oocytes were removed from adult female Xenopus laevis frogs and prepared as described previously (Goldin, 1991) and incubated in ND96 media, which consists of (in $\mathrm{mM}$ ): $96 \mathrm{NaCl}, 2 \mathrm{KCl}, 1.8 \mathrm{CaCl}_{2}, 1 \mathrm{MgCl}_{2}$, and $5 \mathrm{HEPES}, \mathrm{pH} \mathrm{7.5,}$ supplemented with $0.1 \mathrm{mg} / \mathrm{ml}$ gentamycin, $0.55 \mathrm{mg} / \mathrm{ml}$ pyruvate, and 0.5 mM theophylline. Scn8a and Rat2 sodium channel RNA were injected at
$100 \mathrm{pg} /$ oocyte, and Rat1 RNA was injected at $50 \mathrm{ng} /$ oocyte. The oocytes were incubated for $24-48 \mathrm{hr}$ at $20^{\circ} \mathrm{C}$ in ND96.

Sodium currents were recorded using the cut-open oocyte technique (Taglialatela et al., 1992) with the CA-1 high-performance oocyte voltage clamp (Dagan, Minneapolis, MN) and Digidata 1200A interface (Axon Instruments, Foster City, CA) and pCLAMP 6.0.3 software (Axon Instruments), as described previously (Kontis et al., 1997). Temperature was maintained at $20^{\circ} \mathrm{C}$ using a $\mathrm{HCC}-100 \mathrm{~A}$ temperature controller (Dagan). The intracellular solution consisted of (in mM): $88 \mathrm{~K}_{2} \mathrm{SO}_{4}, 10$ EGTA, $10 \mathrm{HEPES}$, and $10 \mathrm{Na}_{2} \mathrm{SO}_{4}, \mathrm{pH} \mathrm{7.5}$, and the extracellular solution consisted of (in mM): 120 sodium MES, 10 HEPES, and 1.8 Ca-MES, pH 7.4. Capacitive transients and leak currents were corrected by $\mathrm{P} / 4$ subtraction. Sodium current amplitudes were between 1 and $5 \mu \mathrm{A}$.

For analysis of recovery from inactivation, voltage dependence of persistent current, and the resurgent current, a two-electrode voltage clamp was used at room temperature as described previously (Patton and Goldin, 1991). Although this voltage clamp does not provide the fast time resolution of the cut-open oocyte clamp, it was used for two reasons. First, the oocytes are more stable over long periods of time, which was essential for analyzing recovery from inactivation. Second, it was possible to subtract comparable records obtained in the presence of $400 \mathrm{~nm}$ TTX to eliminate capacitive and leak currents. TTX subtraction was particularly important when measuring persistent and resurgent currents, because outward currents could easily obscure the small persistent and resurgent currents. The bath solution consisted of ND96.

Data analysis. Analysis was performed using pCLAMP 6.0.3 software (Axon Instruments), Excel 7.0 (Microsoft, Redmond, WA), and Sigmaplot 4.0 (Jandel, San Rafael, CA). Inactivation time constants were determined using the Chebyshev method to fit current traces with a single exponential equation: $I=A_{\text {slow }}{ }^{*} \exp \left[-(t-k) / \tau_{\text {slow }}\right]+C$, or a double exponential equation: $\mathrm{I}=\mathrm{A}_{\text {fast }}{ }^{*} \exp \left[-(\mathrm{t}-\mathrm{K}) / \tau_{\text {fast }}\right]+\mathrm{A}_{\text {slow }} * \exp [-(\mathrm{t}-$ $\left.\mathrm{K}) / \tau_{\text {slow }}\right]+C$, where $I$ is the current, $A_{\text {fast }}$ and $A_{\text {slow }}$ represent the percentage of channels inactivating with time constants $\tau_{\text {fast }}$ and $\tau_{\text {slow }}, K$ is the time shift, and $C$ is the steady-state asymptote. The time shift was manually selected by fitting the traces at the time when the currents were just starting to exponentially decrease. Recovery data were fit using a single, double, or triple exponential equation of the form $I=1-$ $\left[A_{1}{ }^{*} \exp \left(-t / \tau_{1}\right), I=1-\left[A_{1}{ }^{*} \exp \left(-t / \tau_{1}\right)+A_{2}{ }^{*} \exp \left(-t / \tau_{2}\right)\right]\right.$, and $I=1-$ $\left[A_{1}{ }^{*} \exp \left(-t / \tau_{1}\right)+A_{2}{ }^{*} \exp \left(-t / \tau_{2}\right)+A_{3}{ }^{*} \exp \left(-t / \tau_{3}\right)\right]$, where $A_{1}, A_{2}$, and $A_{3}$ are the relative proportions of current recovering with time constants $\tau_{1}, \tau_{2}$, and $\tau_{3}$, and $t$ is the recovery interval.

Conductance values were calculated using the formula $G=I /\left(V-V_{\mathrm{r}}\right)$, where $G$ is conductance, $I$ is current amplitude, $V$ is the depolarized membrane potential, and $V_{\mathrm{r}}$ is the reversal potential. Reversal potentials were individually estimated for each data set by fitting the $I-V$ data with an equation that includes terms for both the voltage dependence of conductance and the driving force: $I=\left[1+\exp \left(-0.03937^{*} z^{*}(V-\right.\right.$ $\left.\left.\left.V_{1 / 2}\right)\right)\right]^{-1 *} \mathrm{~g}^{*}\left(V-V_{\mathrm{r}}\right)$, where $z$ is the apparent gating charge, $g$ is a factor related to the number of channels contributing to the macroscopic current, $V$ is equal to the voltage potential of the pulse, and $V_{1 / 2}$ is the half-maximal voltage. Conductance values were fit with a two-state Boltzmann equation, $G=1 /\left(1+\exp \left[-0.03937^{*} z^{*}\left(V-V^{1 / 2}\right)\right]\right)$, with $z$ equal to the apparent gating charge, $V$ equal to the pulse potential, and $V_{1 / 2}$ equal to the voltage required for half-maximal activation. The voltage dependence of fast inactivation data were fit with a two-state Boltzmann equation, $I=1 /\left(1+\exp \left[\left(V-V^{1 / 2}\right) / a\right]\right)$, with $I$ equal to the current amplitude measured during the test depolarization, $V$ equal to the inactivating depolarization potential, $a$ equal to the slope factor, and $V_{1 / 2}$ equal to the voltage depolarization required for half-maximal inactivation.

\section{RESULTS}

\section{Construction of a full-length cDNA clone encoding the Scn8a sodium channel}

The full-length coding region of Scn8a was amplified and cloned from mouse RNA using RT-PCR, as described in Materials and Methods. The sequence closely matches the previously published sequence for Scn8a (Burgess et al., 1995), except in the region of the I-II linker, and is shown schematically in Figure $1 A$. The sequence that was published by Burgess et al. (1995) contained a short cytoplasmic linker consisting of only 87 amino acids between domains I and II. The sequence that we obtained for the I-II linker contains 333 amino acids (Fig. 1B, GenBank accession 
A

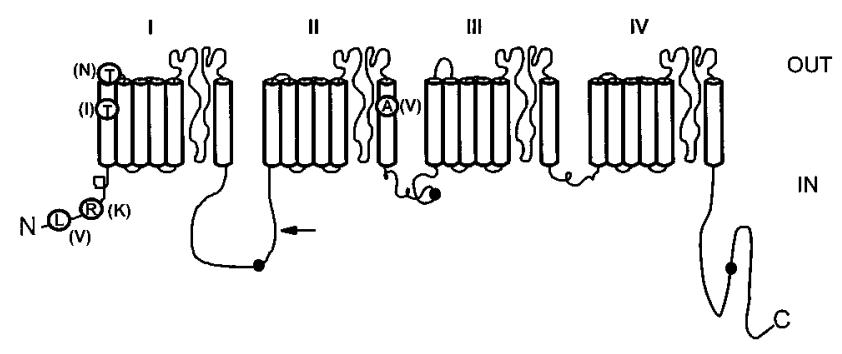

B

IS6

YMIFFVLVIFVGSFYLVNL ILAVVAMAYEEQNQATLEEAEQKEA 430 EFKAMLEQLKKQQEEAQAAAMATSAGTVSEDAIEEEGEDGVGSPRSSSEL 480 SKLSSKSAKERRNRRKKRKQKELSEGEEKGDPEKVFKSESEDGMRRKAFR 530 LPDNR I GRKF SIMNQSLLSI PGSPFLSRHNSKSS IFSFRGPGRFRDPGSE 580 NEFADDEHSTVEESEGRRDSLF IPIRARERRSSYSGYSGYSOCSRSSR IF 630 PSLRRSVKRNSTVDCNGVVSLIGPGSHIGRLLPEVK[DKAATDSATTEVE 670 I KKKGPGSLLVSMEQLASYGRKDR INS IMSVVTNTLVEELEESQRKCPPC 720 WYKFANTFL IWECHPYWI KLKE] VNL I VMDPFVDLA I T I I IVLNTLFMAM 770

IIS1

Figure 1. Diagram of the Scn8a sodium channel. $A$, Diagram of the Scn8a sodium channel, showing the differences between the sequence of the cDNA clone described in this work and the previously published sequence (Burgess et al., 1995). The five amino acid differences are represented by the circled residues, which indicate the sequence of the cDNA clone described here, compared with the originally published sequence shown in parentheses. The positions of the silent nucleotide differences are shown by the solid circles. The position of the 10-amino acid deletion resulting from alternative splicing is shown by the arrow. $B$, Amino acid sequence of the cytoplasmic linker between domains I and II. This sequence was not included in the work by Burgess et al. (1995), but it is identical to the sequence that was later determined for the mouse Scn8a channel by Plummer, Galt, Jones, Burgess, Sprunger, Kohrman, and Meisler (unpublished observations), and it differs at only one position (E in Scn8a compared with D in Rat6 at position 684) compared with the Rat6 (PN4) channel sequence that was determined by Dietrich et al. (1998). The 10 amino acids that are deleted in this spliced form are enclosed in a box.

number AFO49617). This linker sequence is comparable in length to that previously obtained for the Rat6 channel (Schaller et al., 1995) and is identical to the sequence later determined by N. W. Plummer, J. Galt, J. M. Jones, D. L. Burgess, L. K. Sprunger, D. C. Kohrman, and M. H. Meisler (unpublished observations) for the Scn8a channel. In addition, this sequence differs at only one position (E in Scn8a compared with D in Rat6 at position 684) from the sequence for the Rat6 channel (also called PN4) that was determined by Dietrich et al. (1998). Both Plummer, Galt, Jones, Burgess, Sprunger, Kohrman, and Meisler (unpublished observations) and Dietrich et al. (1998) observed different alternatively spliced forms of the I-II linker. The current cDNA clone contains the major form of the transcript, which lacks 10 amino acids following residue 664 in the I-II linker (Fig. $1 A$, arrow, $B$, boxed residues). In addition to the differences in the I-II linker, there are eight nucleotide differences in the remainder of the sequence. Three of the differences are silent and are indicated in Figure $1 A$ by solid circles. Five of the nucleotide differences change the amino acid sequence and are indicated in Figure $1 A$ by the circled residues. Two of the amino acid differences (V5L and K15R) are in the $\mathrm{N}$ terminus, two are in or near domain IS1 (I142T and N153T), and the final difference is in domain IIS6 (V958A).

\section{Functional expression of Scn8a sodium channels and modulation by $\beta_{1}$ and $\beta_{2}$}

Previous studies suggested that Scn8a mediates persistent and resurgent sodium conductances in addition to the transient sodium current, which might reflect unique kinetic or voltagedependent properties for this channel (Raman et al., 1997). To examine this possibility, the functional properties of Scn8a were determined by expression in Xenopus oocytes and compared with those of Rat1 and Rat2 sodium channels. Sodium currents were recorded using the cut-open voltage clamp, which provides sufficient time resolution to accurately compare the inactivation properties of the three channels. Injection of RNA encoding Scn8a resulted in sodium currents that were blocked by TTX with a $K_{\text {app }}$ of $6.4 \pm 0.6 \mathrm{nM}$, which is similar to the values that Smith and Goldin (1998) previously obtained for Rat1 (9.6 $\pm 3.2 \mathrm{~nm})$ and Rat2 $(8.8 \pm 4.0 \mathrm{~nm})$. Figure $2 A$ shows current traces for channels consisting of only the $\alpha$ subunits of Scn8a, Rat1, and Rat2. The records were obtained during depolarizations from -65 to +25 $\mathrm{mV}$ in $10 \mathrm{mV}$ increments from a holding potential of $-100 \mathrm{mV}$. Scn8a channels inactivated more rapidly than either Rat1 or Rat2 channels when only the $\alpha$ subunit was present. Figure $2 B$ shows representative current traces for Scn $8 \mathrm{a}$, Rat1, and Rat $2 \alpha$ subunits coexpressed with the $\beta_{1}$ and $\beta_{2}$ subunits. Coexpression with the two $\beta$ subunits accelerated the inactivation kinetics of all three channels. This has been shown previously to be the case for both Rat1 (Smith and Goldin, 1998) and Rat2 (Isom et al., 1992, 1995; Smith and Goldin, 1998). However, the $\beta$ subunits had less of an effect on Scn8a than on either Rat1 or Rat2, possibly because Scn8a $\alpha$ subunit channels already inactivated faster than either Rat1 or Rat $\alpha$ subunit channels.

\section{Scn8a channels inactivate more rapidly than Rat1 or Rat2 channels}

To quantify the differences in inactivation kinetics between the three channels, the time constants of inactivation were determined for the $\alpha$ subunits alone (Fig. 3A) and for the $\alpha$ subunits coexpressed with the $\beta$ subunits (Fig. $3 B$ ). Currents were recorded during depolarizations from -20 to $+45 \mathrm{mV}$ in $5 \mathrm{mV}$ increments from a holding potential of $-100 \mathrm{mV}$. Inactivation time constants were determined by fitting the current traces with either a single or double exponential equation, as described in Materials and Methods. In Figure 3 the top and middle panels show the slow $\left(\tau_{\text {slow }}\right)$ and fast $\left(\tau_{\text {fast }}\right)$ inactivation time constants, respectively, for Scn8a, Rat1, and Rat2. The bottom panel shows the percent of current inactivating with $\tau_{\text {fast }}$ for each channel. $\tau_{\text {slow }}$ of Scn8a was very similar to $\tau_{\text {slow }}$ for Rat2, and slightly smaller than $\tau_{\text {slow }}$ for Rat1, over the voltage range from +10 to $+45 \mathrm{mV}$. At more negative depolarizations, $\tau_{\text {slow }}$ was similar among the three channels. However, $\tau_{\text {fast }}$ was significantly smaller for Scn8a compared with Rat1 and Rat2. In addition, during depolarizations from -10 to $5 \mathrm{mV}$, Scn8a inactivated with both a fast and slow component, whereas Rat1 and Rat2 inactivated with only a slow component. At more positive depolarizations $(+10$ to $+45 \mathrm{mV}$ ), the percent of current that inactivated with $\tau_{\text {fast }}$ was similar for all three channels.

Coexpression of the $\beta$ subunits did not have a significant effect on $\tau_{\text {slow }}$ of Scn8a compared with $\tau_{\text {slow }}$ of the $\alpha$ subunit alone (Fig. $3 B)$, but it did decrease the magnitude of $\operatorname{Scn} 8 \mathrm{a} \tau_{\text {fast }}$ at the most negative depolarizations ( -25 and $-20 \mathrm{mV}$ ). During depolarizations to $-10 \mathrm{mV}$ or more positive, the $\beta$ subunits increased the percent of Scn8a current inactivating with $\tau_{\text {fast }}$. Although coexpression of the $\beta$ subunits had subtle effects on the kinetics of 
A

\section{$\underline{\text { Scn8a }}$}
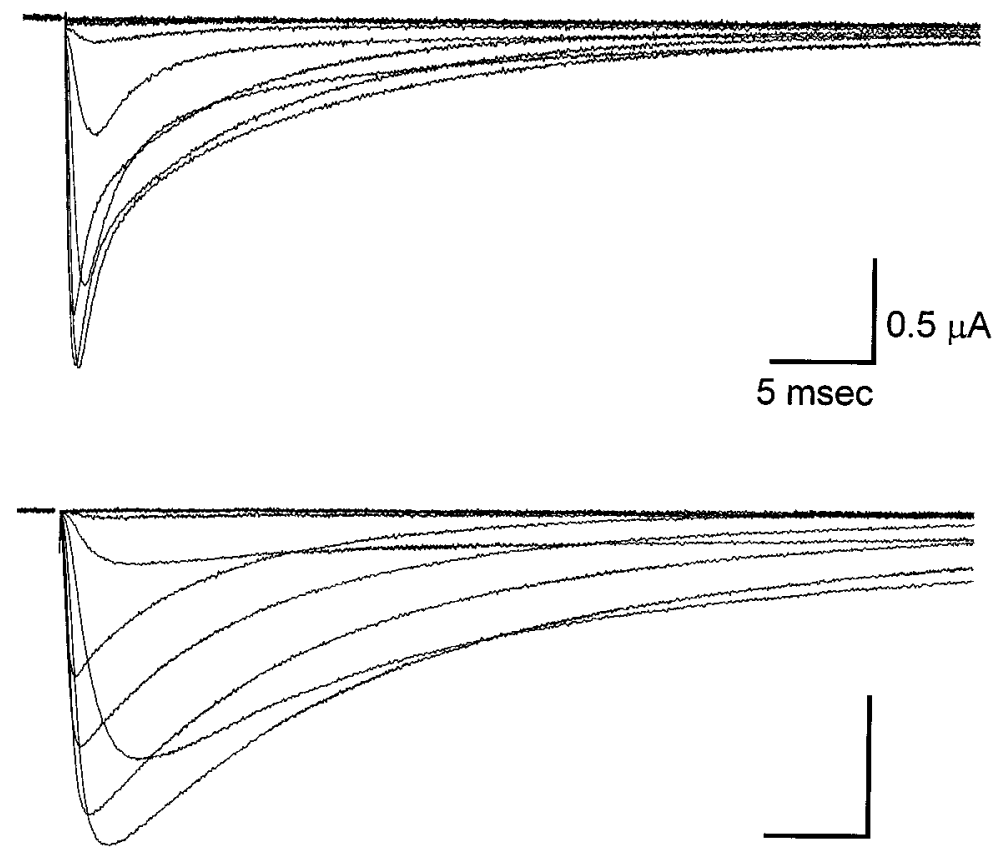

$\underline{\text { Rat2 }}$

$\underline{\text { Rat1 }}$
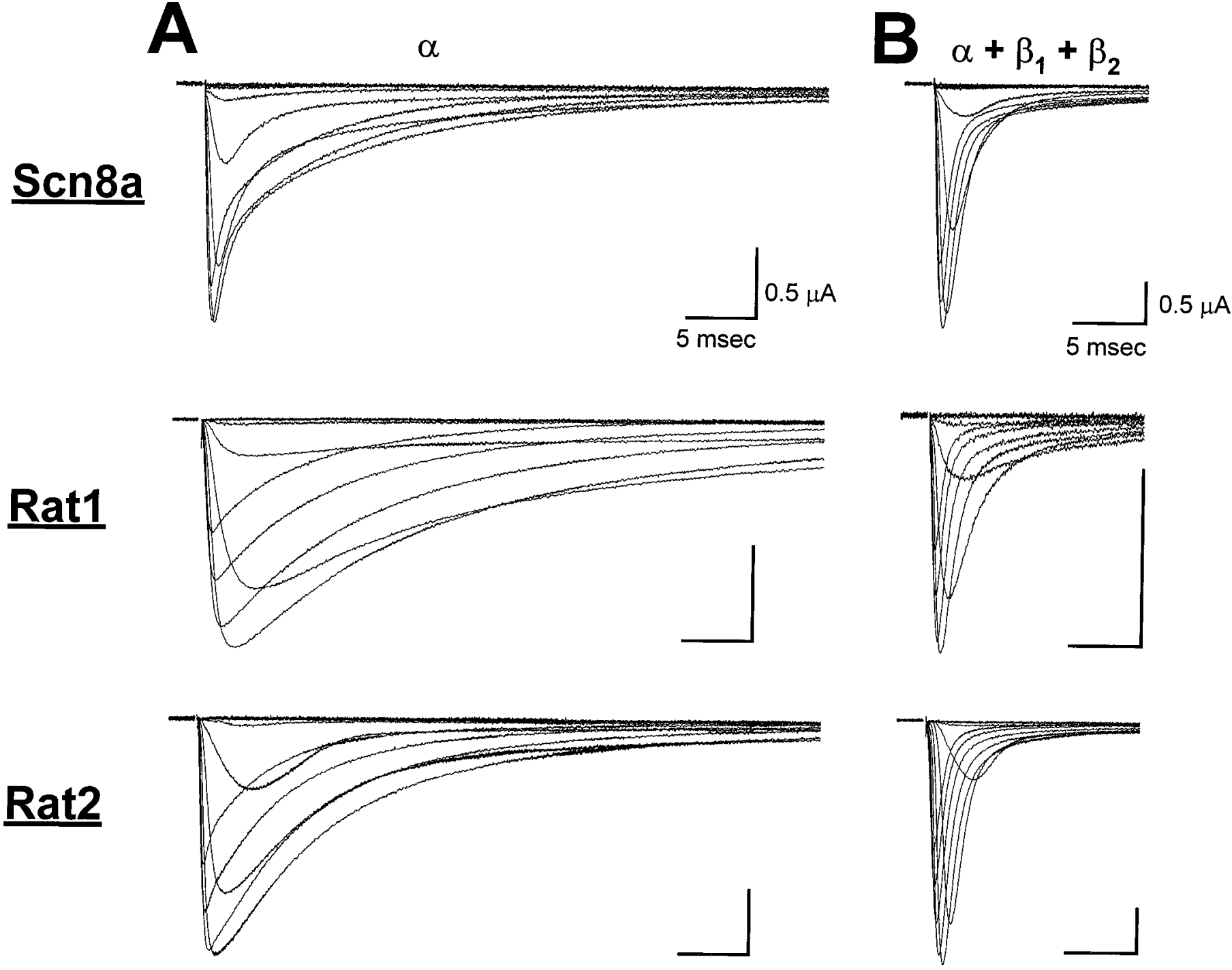

Figure 2. Representative current traces for Scn8a, Rat1, and Rat2. Channels consisting of $\alpha$ subunits alone $(A)$ or $\alpha$ subunits coexpressed with $\beta_{1}$ and $\beta_{2}$ subunits $(B)$ were expressed in Xenopus oocytes as described in Materials and Methods. Currents were recorded using the cut-open oocyte voltage clamp at $20^{\circ} \mathrm{C}$ as described in Materials and Methods. Currents were elicited by membrane depolarizations ranging from -65 to $+25 \mathrm{mV}$ in $10 \mathrm{mV}$ increments from a holding potential of $-100 \mathrm{mV}$. The currents from $\alpha$ subunit channels are shown with a longer time axis to demonstrate inactivation kinetics of these channels, which was much slower than for the channels expressed with the $\beta$ subunits. Calibration: 5 msec, $0.5 \mu \mathrm{A}$.

Scn8a inactivation, it significantly accelerated the kinetics of Rat1 and Rat2 inactivation, so that all three channels inactivated with similar kinetics in the presence of the two $\beta$ subunits.

\section{Recovery from inactivation is initially slower for Scn8a compared with Rat1 and Rat2 sodium channels}

Because $\operatorname{Scn} 8 \mathrm{a} \alpha$ subunit channels inactivated more rapidly than either Rat1 or Rat 2 channels, it was possible that there might also be differences in the kinetics of recovery from inactivation. To examine recovery, currents were recorded using a two-electrode voltage clamp with a protocol consisting of a $50 \mathrm{msec}$ depolarization to $-10 \mathrm{mV}$ to inactivate the channels, followed by a variable recovery interval from 1 to $3000 \mathrm{msec}$ at $-100 \mathrm{mV}$ and a test depolarization to $-10 \mathrm{mV}$ to determine the fraction of current that had recovered. Fractional recovery versus time for Scn8a, Rat1, and Rat $2 \alpha$ subunit channels is shown on a log scale in Figure $4 A$. For the $\alpha$ subunit alone, Scn8a recovery was best fit with a double exponential equation, whereas recovery of Rat1 and Rat2 was best fit with a triple exponential equation. Scn8a initially recovered with a slower time constant (larger $\tau_{1}$ ) compared with Rat1 and Rat2 (Fig. 4A, Table 1). However, Scn8a channels did not demonstrate an intermediate component of recovery. There were only two time constants for recovery of Scn8a channels, and the second time constant $\left(\tau_{2}\right)$ was of the same order of magnitude as $\tau_{3}$ for Rat1 and Rat2 channels. In addition, the percentage of current recovering with the fast time constant was greater for Scn8a than for either Rat1 or Rat2. Therefore, although Scn8a channels initially recovered more slowly than Rat 1 and Rat 2 channels, they recovered more quickly during the interval from 8 to $40 \mathrm{msec}$. The slower time constant for $\operatorname{Scn} 8 \mathrm{a}\left(\tau_{2}\right)$ was larger than $\tau_{3}$ for Rat1 but smaller than $\tau_{3}$ for Rat2.

Fractional recovery for Scn8a, Rat1, and Rat2 channels coexpressed with the $\beta$ subunits is shown versus time on a log scale using a shorter maximum time interval $(300 \mathrm{msec})$ in Figure $4 B$. 


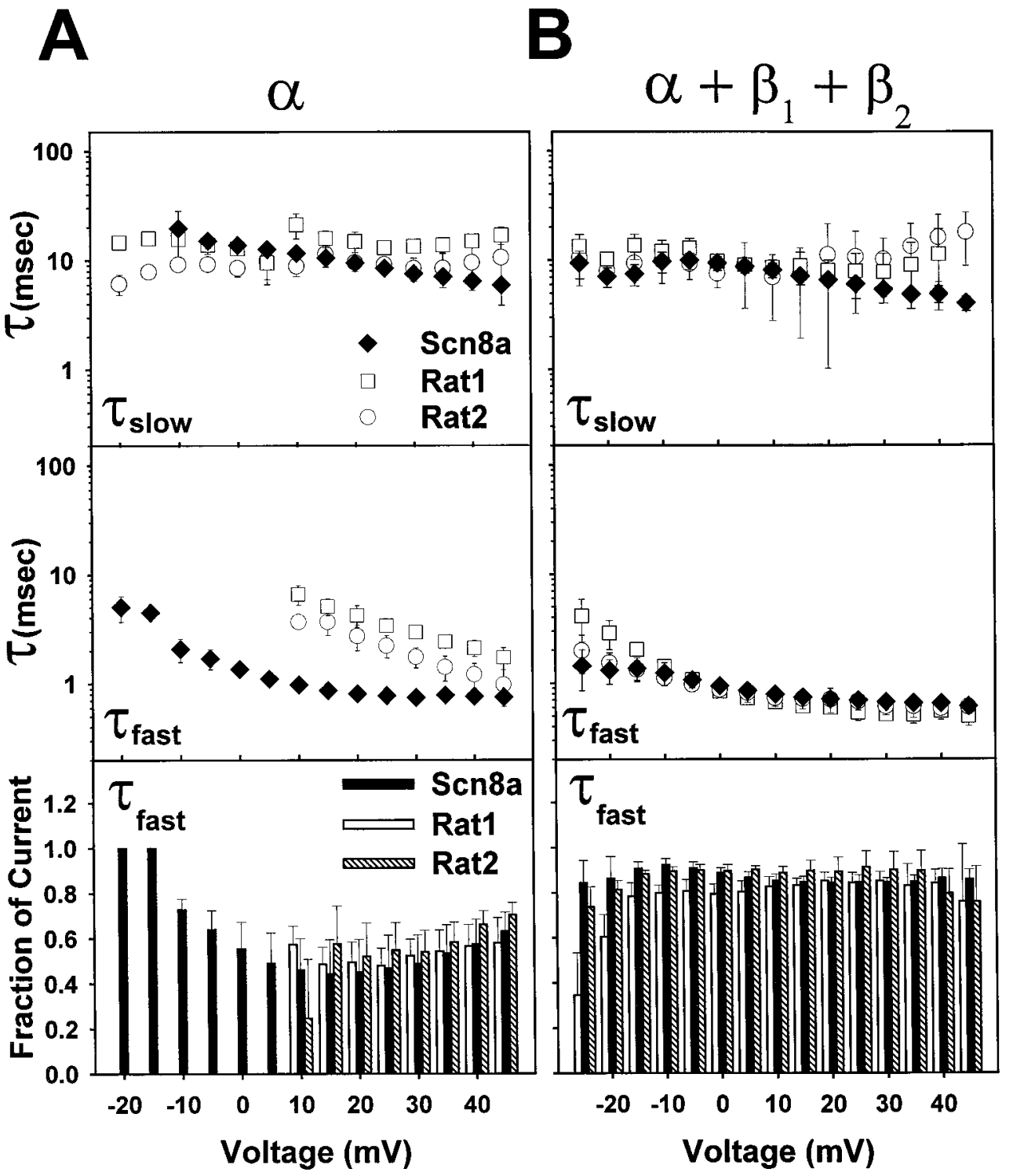

Figure 3. Time constants for fast inactivation of Scn8a, Rat1, and Rat2. Currents were recorded from oocytes expressing Scn8a, Rat1, or Rat2 sodium channels using the cut-open oocyte voltage clamp as described in the legend to Figure 2. The kinetics of inactivation were fit with a single- or doubleexponential equation as described in Materials and Methods, and the time constants representing the slow and fast components are shown on a logarithmic scale in the top and middle panels, respectively, for $\alpha$ subunits alone $(A)$ and $\alpha+\beta_{1}+\beta_{2}(B)$. Scn8a is indicated by the solid diamonds, Rat1 is indicated by open squares, and Rat2 is indicated by open circles. The bottom panel shows the percent of current inactivating with the fast component of inactivation. In all cases, the fraction of $\tau_{\text {fast }}$ plus the fraction of $\tau_{\text {slow }}$ equals 1 . Values represent averages, and error bars indicate SDs. Sample sizes were $\operatorname{Scn} 8$ a $\alpha, n=5$; Rat1 $\alpha, n=7$; Rat2 $\alpha, n=9$; Scn 8 a $\alpha+\beta_{1}+$ $\beta_{2}, n=6$; Rat $1 \alpha+\beta_{1}+\beta_{2}, n=7$; and Rat2 $\alpha+\beta_{1}+\beta_{2}, n=6$.
All of the channels recovered more rapidly in the presence of the $\beta$ subunits. Recovery of Scn8a channels was monoexponential, whereas recovery of Rat 1 and Rat 2 channels was best fit with a double-exponential equation. Initially, Scn8a recovered more slowly than Rat1 and Rat2, which was reflected in a $\tau_{1}$ value that was larger than that for Rat1 or Rat 2 channels (Fig. 4B, Table 1). However, recovery of Scn8a channels was similar or faster than that of Rat1 or Rat 2 channels after a recovery period of $\sim 8 \mathrm{msec}$, because there was no slower time constant of recovery for Scn8a channels.

\section{The voltage dependence of activation is more positive} for Scn8a compared with Rat1 or Rat2

To examine whether Scn8a has unique voltage-dependent properties, the voltage dependence of conductance was measured and fit with a two-state Boltzmann equation, as described in Materials and Methods. When the $\alpha$ subunits were expressed alone, the voltage dependence of conductance for Scn8a had a $V_{1 / 2}$ that was $\sim 9 \mathrm{mV}$ more positive than that of Rat1 or Rat2 (Fig. $5 A$, Table 1 ). There were no significant differences in the slopes of the conductance curves for the three channels. Coexpression of the $\beta$ sub- units with Scn8a resulted in a negative shift of $\sim 9 \mathrm{mV}$ in the $V_{1 / 2}^{1 / 2}$ of conductance (Fig. $5 B$, Table 1). This shift resulted in a $V_{1 / 2}$ for the voltage dependence of conductance that was very similar to that of Rat1 and Rat 2 coexpressed with the $\beta$ subunits, because the $\beta$ subunits shifted the voltage dependence of Scn8a conductance to a greater extent than that of Rat1 and Rat2. In summary, a more positive depolarization was necessary to activate $\operatorname{Scn} 8 \mathrm{a} \alpha$ subunit channels compared with Rat1 or Rat2, whereas the voltage dependence of the three channels was comparable in the presence of the $\beta$ subunits.

\section{The voltage dependence of inactivation is more negative for Scn8a compared with Rat1 and Rat2}

The voltage dependence of steady-state inactivation examines the availability of channels at a given potential, which can have profound effects on the excitability of a cell. The voltage dependence of Scn8a inactivation was determined and compared with that of Rat1 and Rat 2 channels. Scn 8 a $\alpha$ subunit channels demonstrated a $V_{1 / 2}$ for steady-state inactivation that was more negative than that of Rat1 or Rat 2 channels (Fig. $5 C$, Table 1). There were no significant differences in the slopes of the steady-state 
A
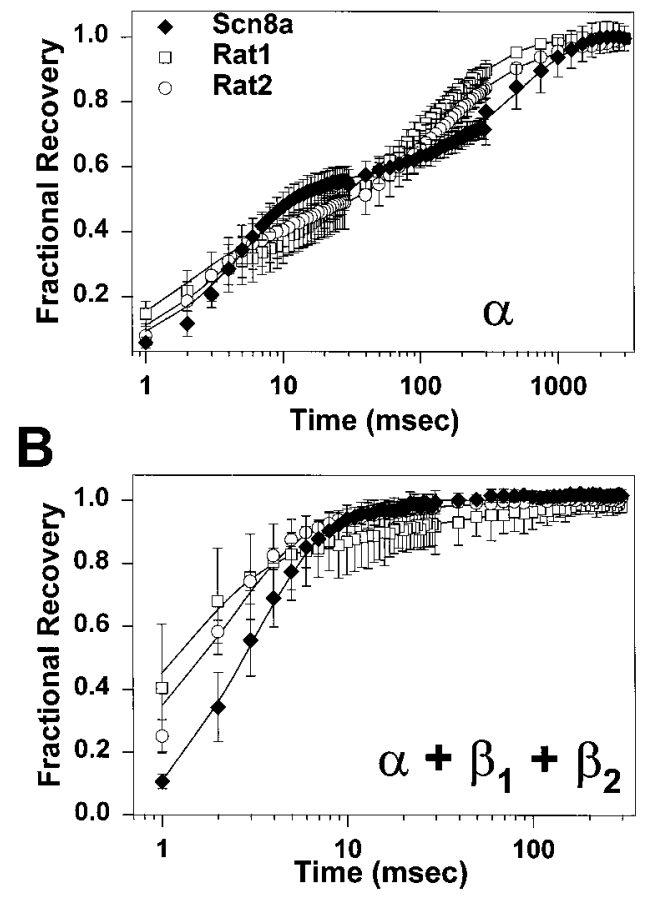

Figure 4. Recovery from fast inactivation of Scn8a, Rat1, and Rat2 sodium currents. Recovery from inactivation was measured with a twoelectrode voltage clamp using a two-pulse protocol consisting of an initial conditioning pulse to $-10 \mathrm{mV}$ for $50 \mathrm{msec}$ (which inactivated $>95 \%$ of the channels), a variable recovery interval, and a test pulse to $-10 \mathrm{mV}$ to measure the amount of current that had recovered. Fractional recovery was calculated by dividing the current amplitude during the test pulse by the amplitude during the corresponding conditioning pulse. Fractional recovery is plotted on a log scale as a function of recovery time for $\alpha$ subunits alone $(A)$ and $\alpha+\beta_{1}+\beta_{2}(B)$. Scn $8 \mathrm{a}$ is indicated by the solid diamonds, Rat1 is indicated by open squares, and Rat 2 is indicated by open circles. Values represent averages, and error bars indicate SDs. The data were fit with a single-, double-, or triple-exponential equation as described in Materials and Methods, and the parameters of the fits and sample sizes are shown in Table 1.

inactivation curves among the three channels. Coexpression of the $\beta$ subunits with Scn8a did not significantly affect the voltage dependence of inactivation (Table 1). In contrast, coexpression of the $\beta$ subunits markedly shifted the $V_{1 / 2}$ of Rat 2 channels in the negative direction, so that the curve for Rat 2 channels with the $\beta$ subunits was more negative than that for Scn8a channels (Fig. $5 D)$. Therefore, $\operatorname{Scn} 8$ a $\alpha$ subunit channels were inactivated at more negative depolarizations than were Rat 1 or Rat 2 channels, but in the presence of the $\beta$ subunits Rat 2 channels were inactivated at more negative potentials than Scn8a channels.

\section{The persistent current of Scn8a increases with} increasing voltage, whereas that of Rat1 decreases

Previous studies have identified persistent and resurgent sodium currents in cerebellar Purkinje neurons (Llinas and Sugimori, 1980; Raman and Bean, 1997). In the rat, these cells have been shown to express Rat1, Rat2, and Rat6, which is the Scn8a ortholog (Black et al., 1994; Felts et al., 1995; Schaller et al., 1995; Vega-Saenz de Miera et al., 1997). It has been suggested that Scn8a (or the orthologous channel) mediates the persistent and resurgent currents (Raman et al., 1997; Vega-Saenz de Miera et al., 1997). Therefore, we examined the level of persistent current for Scn8a compared with that for Rat1 and Rat2 channels. Per- sistent current $\left(I_{\mathrm{pc}}\right)$ was measured $50 \mathrm{msec}$ after the beginning of a depolarization and normalized to the peak current $\left(I_{\text {peak }}\right)$, as shown in Figure $6 A$ for Scn8a $+\beta_{1}+\beta_{2}$ during a depolarization to $+25 \mathrm{mV}$. To eliminate any contamination from other conductances, all analyses were performed on current records from which had been subtracted comparable records obtained in the presence of TTX. The levels of persistent current for Scn8a, Rat1, and Rat 2 channels coexpressed with the $\beta$ subunits were examined over a voltage range from -20 to $+25 \mathrm{mV}$ (Fig. 6B). Rat 2 demonstrated a very small persistent current over the entire voltage range. The percent of persistent current for Rat1 decreased with more positive depolarizations. In contrast, the percent of persistent current for Scn8a increased with more positive depolarizations. Therefore, Rat1 demonstrated a greater percent of persistent current at negative potentials $(-20 \mathrm{mV})$, and Scn8a demonstrated a greater percent of persistent current at positive potentials $(\geq 0 \mathrm{mV})$. These data indicate that Scn8a channels mediate a persistent sodium current that could be significant during the firing of an action potential.

\section{Does Scn8a mediate resurgent current?}

Raman and Bean (1997) and Raman et al. (1997) identified a small transient current in Purkinje neurons that is elicited by an action potential-like waveform. This current was termed a resurgent current, and it was not present in Purkinje neurons from Scn8a null mice. To examine the possibility that this current is an inherent property of the Scn8a channel, we used a similar protocol to record from oocytes expressing Scn8a, Rat1, or Rat2 $\alpha$ subunits coexpressed with the $\beta$ subunits. Because the magnitude of the resurgent current was quite small $(\sim 4 \%)$ compared with the peak current in the studies by Raman and Bean (1997) and Raman et al. (1997), we used oocytes expressing very high levels of peak sodium current $(8-30 \mu \mathrm{A})$. Oocytes were depolarized to $+30 \mathrm{mV}$ for $48 \mathrm{msec}$ from a holding potential of $-90 \mathrm{mV}$, which resulted in a steady-state level of inactivation. This depolarization was immediately followed by test depolarizations ranging from -80 to $+40 \mathrm{mV}$ in $10 \mathrm{mV}$ increments to elicit any resurgent current (Fig. 7A). Figure $7 B$ shows representative current traces for Scn8a currents during test depolarizations from -60 to +40 $\mathrm{mV}$ in $20 \mathrm{mV}$ increments, after subtraction of comparable records obtained in the presence of TTX. At negative depolarizations, there was an initial rapidly decaying current followed by a persistent current. At depolarizations above $+20 \mathrm{mV}$, the transient current was less pronounced.

Figure $7 C$ shows the percent of the maximal transient current relative to the peak current obtained during a single depolarization to $0 \mathrm{mV}$ ( $-10 \mathrm{mV}$ for Rat1 and Rat2). Scn8a had a much larger percent of transient current compared with Rat1 or Rat2. The level of transient current for $\operatorname{Scn} 8$ a was $\sim 4 \%$ of the peak current. This level of current is similar to the level of resurgent current observed in Purkinje cells by Raman and Bean (1997) and Raman et al. (1997). The resurgent current described by Raman et al. (1997) differed in voltage dependence compared with the initial current, with a peak at $-40 \mathrm{mV}$ for the resurgent current compared with $0 \mathrm{mV}$ for the initial current. Therefore, we examined the voltage dependence of the transient current by normalizing the current at each depolarization to the maximal transient current (Fig. 7D). The transient current for all three channels showed similar voltage dependence to the voltage dependence of the peak current, with a peak at $0 \mathrm{mV}$ for $\operatorname{Scn} 8 \mathrm{a}$ and $-10 \mathrm{mV}$ for Rat1 and Rat2. Therefore, although the magnitude of the transient current was similar to that previously observed for resurgent 
Table 1. Parameters of the voltage dependence of activation and inactivation and recovery from inactivation

\begin{tabular}{|c|c|c|c|c|c|c|c|c|c|c|c|c|c|}
\hline \multirow[b]{3}{*}{ Channel } & \multirow{2}{*}{\multicolumn{2}{|c|}{ Activation }} & \multirow[b]{3}{*}{$\mathrm{n}$} & \multirow{2}{*}{\multicolumn{3}{|c|}{ Inactivation }} & \multicolumn{7}{|c|}{ Recovery from Inactivation } \\
\hline & & & & & & & \multicolumn{2}{|l|}{$\tau_{1}$} & \multicolumn{2}{|l|}{$\underline{\tau_{2}}$} & \multicolumn{2}{|l|}{$\tau_{3}$} & $n$ \\
\hline & $\mathrm{V}_{1 / 2}(\mathrm{mV})$ & $z\left(e_{o}\right)$ & & $\mathrm{V}_{1 / 2}(\mathrm{mV})$ & $\mathrm{a}(\mathrm{mV})$ & $n$ & msec & $\%$ & msec & $\%$ & msec & $\%$ & \\
\hline $\operatorname{Sen} 8 \mathrm{a}+\beta_{1}+\beta_{2}$ & $-17 \pm 4$ & $4.2 \pm 0.8$ & 6 & $-51 \pm 1$ & $4.9 \pm 0.2$ & 6 & $3.8 \pm 1.0$ & 100 & $\mathrm{ND}^{b}$ & $\mathrm{ND}^{b}$ & $\mathrm{ND}^{b}$ & $\mathrm{ND}^{b}$ & 5 \\
\hline Rat1 & $-17 \pm 4$ & $4.8 \pm 0.5$ & 8 & $-35 \pm 2$ & $5.2 \pm 0.5$ & 6 & $1.9 \pm 0.6$ & $34 \pm 4$ & $46 \pm 27$ & $30 \pm 12$ & $280 \pm 130$ & $36 \pm 13$ & 4 \\
\hline $\operatorname{Rat} 1+\beta_{1}+\beta_{2}$ & $-15 \pm 2$ & $3.9 \pm 0.4$ & 6 & $-41 \pm 2$ & $4.8 \pm 0.6$ & 3 & $1.4 \pm 0.6$ & $82 \pm 1$ & $13 \pm 4$ & $8.6 \pm 4.0$ & $\mathrm{ND}^{c}$ & $\mathrm{ND}^{c}$ & 4 \\
\hline $\operatorname{Rat} 2+\beta_{1}+\beta_{2}$ & $-22 \pm 4$ & $4.5 \pm 0.4$ & 5 & $-57 \pm 4$ & $4.5 \pm 0.3$ & 5 & $2.2 \pm 0.2$ & $93 \pm 1$ & $47 \pm 44$ & $3.5 \pm 2.3$ & $\mathrm{ND}^{d}$ & $\mathrm{ND}^{d}$ & 8 \\
\hline
\end{tabular}

${ }^{a} \mathrm{ND}$, not determined because recovery for these channels was best fit with a double-exponential equation.

${ }^{b} \mathrm{ND}$, not determined because recovery for these channels was best fit with a single-exponential equation.

${ }^{c} \mathrm{ND}$, not determined because the time constant was $>1.5 \mathrm{sec}$ and could not be accurately quantified.

${ }^{d} \mathrm{ND}$, not determined because the percentage of current recovering with $\tau_{3}$ was $<4 \%$.
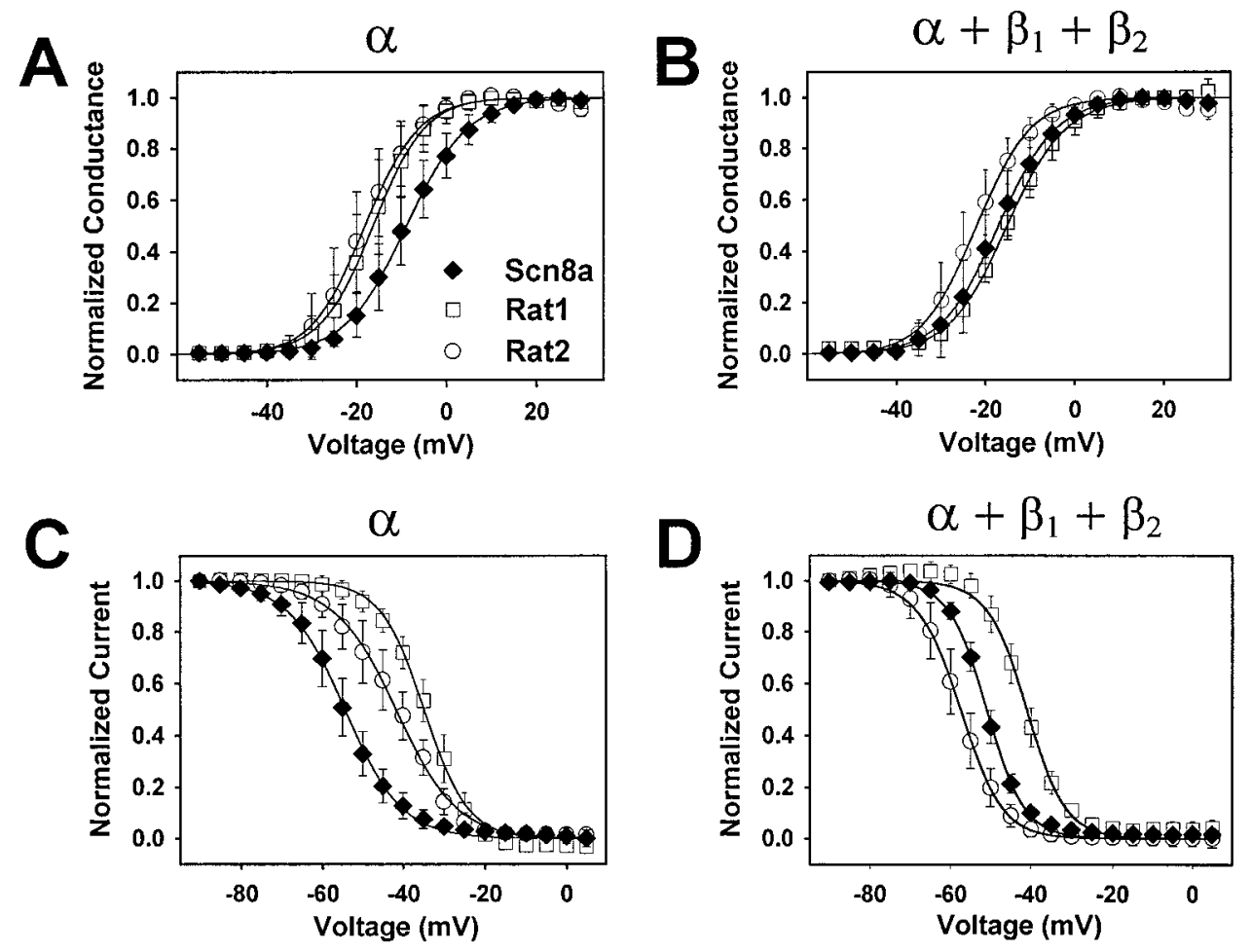

Figure 5. Voltage dependence of activation and inactivation for Scn8a, Rat1, and Rat2 sodium channels. The voltage dependence of activation is shown for $\alpha$ subunits (A) alone and $\alpha+\beta_{1}+\beta_{2}(B)$. Sodium currents were recorded using a cut-open oocyte clamp and elicited by depolarizing pulses from a holding potential of $-100 \mathrm{mV}$ to potentials ranging from -55 to $+30 \mathrm{mV}$ in $5 \mathrm{mV}$ increments. Conductance values were calculated by dividing the peak current amplitude by the driving force at each potential and normalizing to the maximum conductance, as described in Materials and Methods. Scn8a is indicated by the solid diamonds, Rat1 is indicated by open squares, and Rat 2 is indicated by open circles. Values represent averages, and error bars indicate SDs. The data were fit with a two-state Boltzmann equation as described in Materials and Methods, and the parameters of the fits and sample sizes are shown in Table 1 . The voltage dependence of inactivation is shown for $\alpha$ subunits alone $(C)$ and $\alpha+\beta_{1}$ $+\beta_{2}(D)$. The voltage dependence of inactivation was determined using a two-step protocol in which a $500 \mathrm{msec}$ conditioning pulse to potentials ranging from -90 to +5 $\mathrm{mV}$ was followed by a $25 \mathrm{msec}$ test pulse to $-10 \mathrm{mV}$ to measure peak current amplitude. The peak current amplitude during the test pulse was normalized to the maximum current amplitude and is plotted as a function of the conditioning pulse potential. Scn8a is indicated by the solid diamonds, Rat1 is indicated by open squares, and Rat 2 is indicated by open circles. Values represent averages, and error bars indicate SDs. The data were fit with a two-state Boltzmann equation as described in Materials and Methods, and the parameters of the fits and sample sizes are shown in Table 1.

current, the fact that the voltage dependence was equivalent to that of the peak current suggests that none of these channels mediates a true resurgent current when expressed in Xenopus oocytes.

\section{DISCUSSION}

We have constructed a full-length cDNA clone encoding the Scn8a sodium channel. Injection of RNA transcribed from this clone resulted in functional sodium currents in Xenopus oocytes. There were five amino acid differences between the sequence of the clone that we obtained and the sequence previously determined for Scn8a (Burgess et al., 1995) (Plummer, Galt, Jones, Burgess, Sprunger, Kohrman, and Meisler, unpublished observations). One or more of the first four differences might represent polymorphisms, because our data were obtained from inbred
C57BL/6J mice, and the first 257 residues reported by Burgess et al. (1995) were obtained from strain ICR mice. Consistent with this hypothesis, the leucine that we noted at position 5 is present in the Rat6 sequence that was determined by Dietrich et al. (1998) and in mouse strain DBA/2 (Kohrman et al., 1996b). However, it is possible that at least some of the differences represent artifacts of the PCR. The cytoplasmic linker connecting domains I-II in the cDNA clone that we constructed is 10 amino acids shorter than the longest alternatively spliced form that was detected in mice (Plummer, Galt, Jones, Burgess, Sprunger, Kohrman, and Meisler, unpublished observations) and rat (Schaller et al., 1995; Dietrich et al., 1998). This shorter form represents the major form of the Scn8a transcript in the brains of mice (Plummer, Galt, Jones, Burgess, Sprunger, Kohrman, and 
A

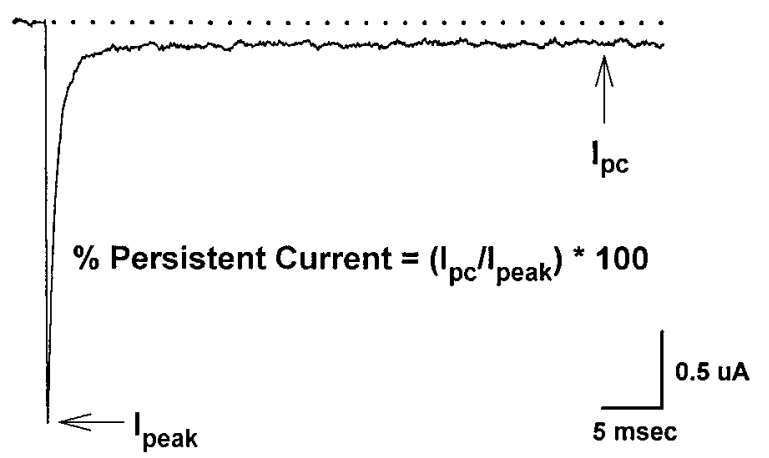

B

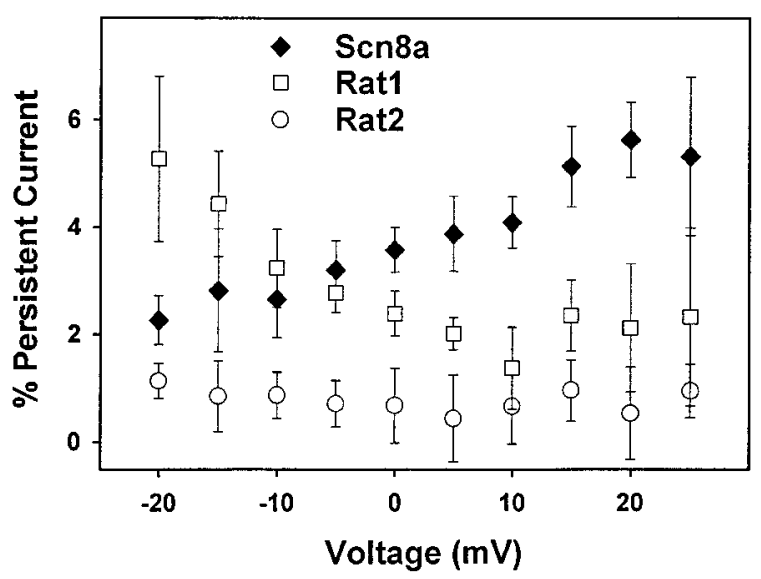

Figure 6. Voltage dependence of persistent current for Scn8a, Rat1, and Rat2 sodium channels. $A$, Representative current trace using a twoelectrode voltage clamp of $\operatorname{Scn} 8 \mathrm{a}+\beta_{1}+\beta_{2}$ channels during a depolarization to $+25 \mathrm{mV}$. The percent of persistent current was calculated from TTX-subtracted current traces by measuring the persistent current remaining at $50 \mathrm{msec}\left(I_{\mathrm{pc}}\right)$ and dividing by the peak current $\left(I_{\text {peak }}\right)$ for each depolarization. $B$, The percent of persistent current is shown for Scn8a (solid diamonds), for Rat1 (open squares), and for Rat2 (open circles) over a voltage range from -20 to $+25 \mathrm{mV}$ in $5 \mathrm{mV}$ increments. Channels were coexpressed with the $\beta_{1}$ and $\beta_{2}$ subunits. Values represent averages, and error bars indicate SDs. Sample sizes were Scn8a, $n=5$; Rat1, $n=5$; and Rat2, $n=5$.

Meisler, unpublished observations) and rat (Dietrich et al., 1998). The position and size of this variation corresponds to alternatively spliced forms that were described previously for Rat1 (Schaller et al., 1992).

The electrophysiological properties of the Scn8a sodium channel differed somewhat from those determined by Dietrich et al. (1998) for the Rat6 (PN4) channel. Specifically, Dietrich et al. (1998) observed slightly faster inactivation kinetics and significantly more negative $V_{1 / 2}^{1 / 2}$ values for activation and inactivation. At least some of these differences probably result from different recording techniques, because Dietrich et al. (1998) used macropatch recording and we used the cut-open oocyte voltage clamp. Some of the electrophysiological properties that we observed for the Scn8a sodium channel also differed from those of the Rat1 and Rat 2 channels, which are expressed in the adult CNS. The most dramatic difference was that $\operatorname{Scn} 8 \mathrm{a} \alpha$ subunit channels inactivated more rapidly than either Rat1 or Rat $2 \alpha$ subunit channels. With respect to voltage dependence, Scn8a channels activated at more positive potentials than Rat 1 and Rat 2 but demonstrated a more negative $V_{1 / 2}$ for steady-state inactivation. Based on these characteristics alone, cells expressing only Scn8a should have decreased electrical excitability and a higher threshold for activation of action potentials compared with cells expressing Rat1 or Rat2.

However, sodium channels in the CNS are most likely associated with one or both of the $\beta$ subunits, which modulate the properties of the Scn8a sodium channel. The $\beta$ subunits accelerated the kinetics of inactivation, but to much less of an extent than was observed for Rat1 and Rat2, so that all three channels demonstrated similar kinetics in the presence of the $\beta$ subunits. Coexpression of the $\beta$ subunits caused a large hyperpolarizing shift in the voltage dependence of activation for Scn8a but had no significant effect on the voltage dependence of steady-state inactivation. Recovery from inactivation was accelerated by the presence of the $\beta$ subunits. Because of these effects, the electrophysiological properties of Scn8a sodium channels were very similar to those of Rat1 and Rat 2 sodium channels when all three channels were coexpressed with the $\beta$ subunits.

We observed that $\operatorname{Scn} 8 \mathrm{a}$ with the $\beta$ subunits demonstrated a persistent current that increased linearly with more positive membrane potentials. In contrast, Rat1 demonstrated a persistent current that was large at negative potentials and decreased with more positive membrane potentials. These results suggest that Scn8a channels mediate a persistent current that is largest when an action potential is fired, which might play a critical role in the repetitive firing of action potentials seen in Purkinje neurons. However, the persistent current from Rat1 may also be involved in the excitability of Purkinje neurons.

Raman et al. (1997) observed a persistent current in normal Purkinje cells, and the magnitude of that current was greatly decreased in cells from mice containing a Scn8a null mutation. Vega-Saenz de Miera et al. (1997) used single-cell RT-PCR from guinea pig cerebellar slices and in situ hybridization to show that Rat6 (the Scn8a ortholog) and Rat1 are expressed in Purkinje neurons. There was no detectable level of Rat 2 mRNA in the studies by Vega-Saenz de Miera et al. (1997), although Rat2 mRNA was detected in Purkinje neurons by Black et al. (1994). Based on these results, Vega-Saenz de Miera et al. (1997) suggested that Rat1 mediates a transient current in Purkinje neurons, whereas Scn8a mediates noninactivating persistent current in Purkinje neurons. Our data and the results of Raman et al. (1997) support a role for Rat1 and Scn8a contributing to both transient and persistent currents.

In addition to the persistent current, another unique sodium conductance termed a resurgent current has been observed in cerebellar Purkinje neurons (Raman and Bean, 1997; Raman et al., 1997). The resurgent current is a small sodium current that is elicited by a waveform that simulates an action potential. This current was observed both in normal Purkinje cells and in cells containing the null Scn8a mutation, but it was much larger in the normal Purkinje cells expressing Scn8a. These results suggested that Scn8a might be the primary sodium channel responsible for the resurgent current. Using similar electrophysiological protocols to elicit resurgent current, we observed a current that was $\sim 4 \%$ of the peak transient current. However, that current inactivated with a time course similar to that of the initial transient current. In addition, the current that we observed demonstrated a peak amplitude at $0 \mathrm{mV}$, whereas the resurgent current in Pur- 

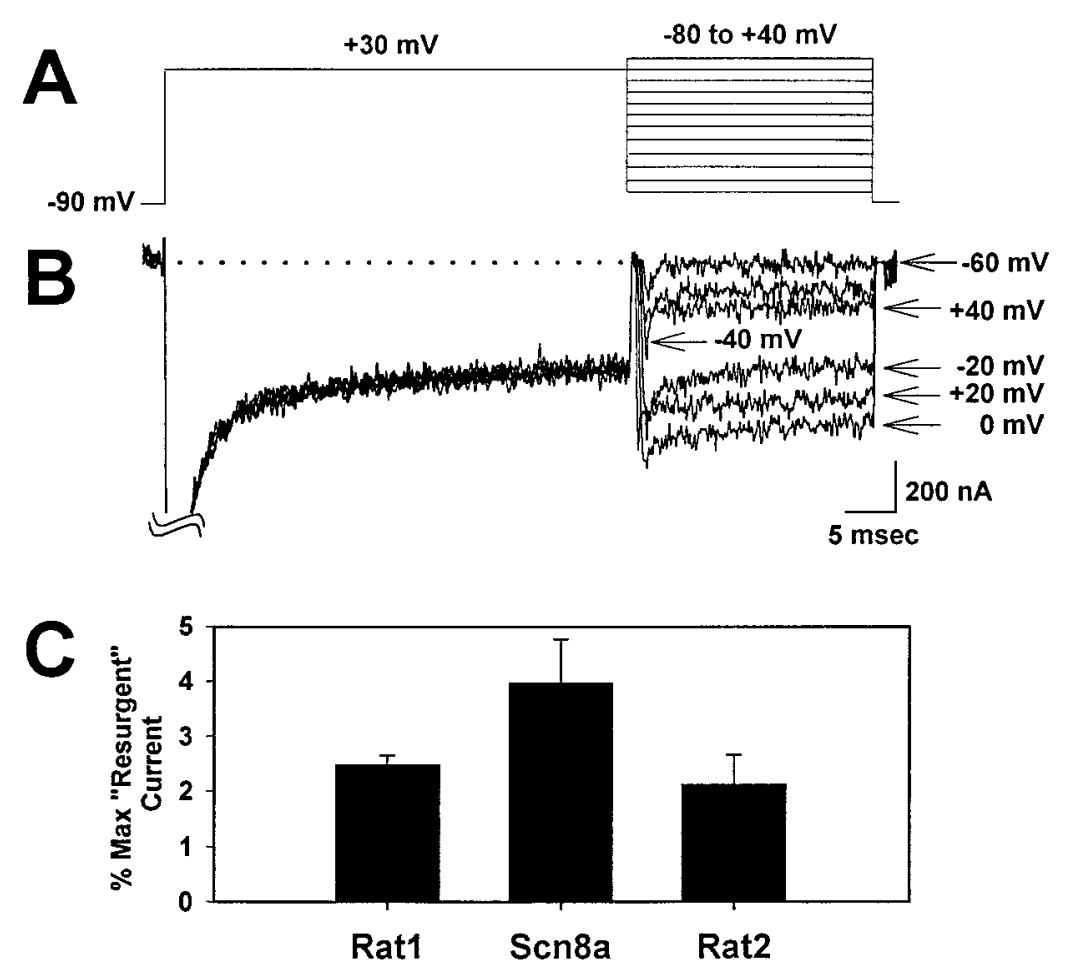

D

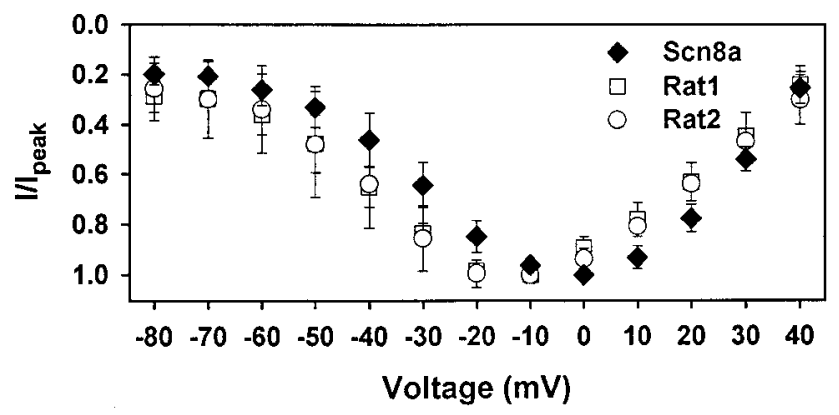

kinje cells demonstrated a peak at approximately $-40 \mathrm{mV}$. Finally, currents from Rat1 and Rat 2 channels demonstrated similar "resurgent" currents, although the levels were approximately half of that seen for Scn8a. It seems likely that the current that we observed was not a true resurgent current but resulted from the large persistent current observed for $\operatorname{Scn} 8$ a at positive depolarizations. With a large persistent current at $+30 \mathrm{mV}$, depolarization to a more negative potential would increase the driving force for sodium and result in a significant increase in current amplitude. Although the current that we identified was most likely not the resurgent current, it may contribute to the resurgent current in Purkinje cells, particularly because the magnitude was similar to that observed in Purkinje cells.

There are several possible reasons why we did not observe a true resurgent current from Scn8a channels expressed in Xenopus oocytes. First, it is possible that the Scn8a channel undergoes post-translational modification in Purkinje neurons, which may change some of the electrophysiological properties of the channel. Second, there may be accessory proteins other than the $\beta$ subunits in Purkinje neurons, and these could alter the properties of the channel. For example, Ma et al. (1997) have shown that the Rat2 channel mediates persistent current if that channel is coexpressed with G-protein $\beta \gamma$ subunits. A variation of this explanation is that the $\beta$ subunits prevent resurgent current. However, we did not
Figure 7. Possible resurgent current resulting from Scn8a, Rat1, and Rat2 channels. Channels were coexpressed with the $\beta$ subunits as described in the legend to Figure 2. Oocytes expressing current levels of $8-30 \mu \mathrm{A}$ were used to attain larger and more easily measurable potential resurgent currents, which were recorded using a two-electrode voltage clamp. $A$, The protocol used to elicit resurgent current consists of depolarization from a holding potential of $-90 \mathrm{mV}$ to a membrane depolarization of $+30 \mathrm{mV}$ for $48 \mathrm{msec}$ followed by a range of depolarizations from -80 to $+50 \mathrm{mV}$ in $10 \mathrm{mV}$ increments for $25 \mathrm{msec}$. $B$, Representative TTX subtracted current traces are shown for Scn8a channels during depolarizations from -60 to $+40 \mathrm{mV}$ in $20 \mathrm{mV}$ increments. The vertical scale bar corresponds to $10 \%$ of the initial current evoked during the depolarization to $+30 \mathrm{mV}$, which is off the scale. $C$, The percent of the peak potential resurgent current normalized to the peak initial current is shown. $D$, The potential resurgent currents during each depolarization were normalized to the peak current and plotted versus voltage for Scn8a (solid diamonds), Rat1 (open squares), and Rat2 (open circles). Values represent averages, and error bars indicate SDs. Sample sizes were Scn8a, $n=4$; Rat1, $n=5$; and Rat2, $n=4$.

observe any resurgent current when the Scn8a channel was expressed in the absence of the $\beta$ subunits (data not shown). Third, it is possible that multiple different sodium channels have to be expressed to obtain resurgent current. We consider this explanation unlikely, because we have not observed resurgent current when Scn8a was coexpressed with Rat1 and/or Rat 2 in oocytes (data not shown). Finally, it is possible that the resurgent current may be a property of one specific splice variant of the Scn8a sodium channel. Plummer, Galt, Jones, Burgess, Sprunger, Kohrman, and Meisler (unpublished observations) have shown that multiple splice variants of the $\operatorname{Sen} 8 \mathrm{a} \alpha$ subunit exist. This hypothesis can now be tested by constructing and expressing the various splice variants.

\section{REFERENCES}

Auld VJ, Goldin AL, Krafte DS, Marshall J, Dunn JM, Catterall WA, Lester HA, Davidson N, Dunn RJ (1988) A rat brain $\mathrm{Na}^{+}$channel $\alpha$ subunit with novel gating properties. Neuron 1:449-461.

Beckh S, Noda M, Lübbert H, Numa S (1989) Differential regulation of three sodium channel messenger RNAs in the rat CNS during development. EMBO J 8:3611-3636.

Black JA, Yokoyama S, Higashida H, Ransom BR, Waxman SG (1994) Sodium channel mRNAs I, II, and III in the CNS: cell-specific expression. Mol Brain Res 22:275-289.

Burgess DL, Kohrman DC, Galt J, Plummer NW, Jones JM, Spear B, 
Meisler MH (1995) Mutation of a new sodium channel gene, $S c n 8 a$, in the mouse mutant "motor endplate disease." Nat Genet 10:461-465.

Catterall WA (1992) Cellular and molecular biology of voltage-gated sodium channels. Physiol Rev 72:S15-S48.

Dick DJ, Boakes RJ, Harris JB (1985) A cerebellar abnormality in the mouse with motor end-plate disease. Neuropathol Appl Neurobiol 11:141-147.

Dietrich PS, McGivern JG, Delgado SG, Koch BD, Eglen RM, Hunter JC, Sangameswaran L (1998) Functional analysis of a voltage-gated sodium channel and its splice variant from rat dorsal root ganglion. J Neurochem 70:2262-2272.

Felts PA, Black JA, Waxman SG (1995) Expression of sodium channel $\alpha$ - and $\beta$-subunits in the nervous system of the myelin-deficient rat. J Neurocytol 24:654-666.

Felts PA, Yokoyama S, Dib-Hajj S, Black JA, Waxman SG (1997) Sodium channel $\alpha$-subunit mRNAs I, II, III, NaG, Na6, and hNE (PN1): different expression patterns in developing rat nervous system. Mol Brain Res 45:71-82.

Furuyama T, Morita Y, Inagaki S, Takagi H (1993) Distribution of I, II, and III subtypes of voltage-sensitive $\mathrm{Na}^{+}$channel mRNA in the rat brain. Mol Brain Res 17:169-173.

Garcia KD, Sprunger LK, Meisler MH, Beam KG (1998) The sodium channel $S c n 8 a$ is the major contributor to the postnatal developmental increase of sodium current density in spinal motoneurons. J Neurosci 14:5234-5239.

Goldin AL (1991) Expression of ion channels by injection of mRNA into Xenopus oocytes. Methods Cell Biol 36:487-509.

Gordon D, Merrick D, Auld V, Dunn R, Goldin AL, Davidson N, Catterall WA (1987) Tissue-specific expression of the $R_{I}$ and $R_{I I}$ sodium channel subtypes. Proc Natl Acad Sci USA 84:8682-8686.

Harris JB, Boakes RJ, Court JA (1992) Physiological and biochemical studies on the cerebellar cortex of the murine mutants "jolting" and "motor end-plate disease". J Neurol Sci 110:186-194.

Isom LL, DeJongh KS, Patton DE, Reber BFX, Offord J, Charbonneau H, Walsh K, Goldin AL, Catterall WA (1992) Primary structure and functional expression of the $\beta_{1}$ subunit of the rat brain sodium channel. Science 256:839-842.

Isom LL, Ragsdale DS, De Jongh KS, Westenbroek RE, Reber BFX, Scheuer T, Catterall WA (1995) Structure and function of the $\beta_{2}$ subunit of brain sodium channels, a transmembrane glycoprotein with a CAM motif. Cell 83:433-442.

Joho RH, Moorman JR, VanDongen AMJ, Kirsch GE, Silberberg H, Schuster G, Brown AM (1990) Toxin and kinetic profile of rat brain type III sodium channel expressed in Xenopus oocytes. Mol Brain Res 7:105-113.

Kayano T, Noda M, Flockerzi V, Takahashi H, Numa S (1988) Primary structure of rat brain sodium channel III deduced from the cDNA sequence. FEBS Lett 228:187-194.

Kohrman DC, Plummer NW, Schuster T, Jones JM, Jang W, Burgess DL, Galt J, Spear BT, Meisler MH (1995) Insertional mutation of the motor endplate disease (med) locus on mouse chromosome 15. Genomics 26:171-177.

Kohrman DC, Smith MR, Goldin AL, Harris J, Meisler MH (1996a) A missense mutation in the sodium channel Scn8a is responsible for cerebellar ataxia in the mouse mutant jolting. J Neurosci 16:5993-5999.

Kohrman DC, Harris JB, Meisler MH (1996b) Mutation detection in the med and med $^{\mathrm{J}}$ alleles of the sodium channel Scn8a. J Biol Chem 271:17576-17581.

Kontis KJ, Rounaghi A, Goldin AL (1997) Sodium channel activation gating is affected by substitutions of voltage sensor positive charges in all four domains. J Gen Physiol 110:391-401.

Llinas R, Sugimori M (1980) Electrophysiological properties of in vitro Purkinje cell somata in mammalian cerebellar slices. J Physiol (Lond) 305:171-195.

Ma JY, Catterall WA, Scheuer T (1997) Persistent sodium currents through brain sodium channels induced by G-protein $\beta \gamma$ subunits. Neuron 19:443-452.

Meisler MH, Sprunger LK, Plummer NW, Escayg A, Jones JM (1997) Ion channel mutations in mouse models of inherited neurological diseases. Ann Med 29:569-574.

Noda M, Ikeda T, Kayano T, Suzuki H, Takeshima H, Kurasaki M, Takahashi H, Numa S (1986) Existence of distinct sodium channel messenger RNAs in rat brain. Nature 320:188-192.

Patton DE, Goldin AL (1991) A voltage-dependent gating transition induces use-dependent block by tetrodotoxin of rat IIA sodium channels expressed in Xenopus oocytes. Neuron 7:637-647.

Plummer NW, McBurney MW, Meisler MH (1997) Alternative splicing of the sodium channel Scn8a predicts a truncated two-domain protein in fetal brain and non-neuronal cells. J Biol Chem 272:24008-24015.

Raman IM, Bean BP (1997) Resurgent sodium current and action potential formation in dissociated cerebellar Purkinje neurons. J Neurosci 17:4517-4526.

Raman IM, Sprunger LK, Meisler MH, Bean BP (1997) Altered subthreshold sodium currents and disrupted firing patterns in Purkinje neurons of Scn8a mutant mice. Neuron 19:881-891.

Schaller KL, Krzemien DM, McKenna NM, Caldwell JH (1992) Alternatively spliced sodium channel transcripts in brain and muscle. J Neurosci 12:1370-1381.

Schaller KL, Krzemien DM, Yarowsky PJ, Krueger BK, Caldwell JH (1995) A novel, abundant sodium channel expressed in neurons and glia. J Neurosci 15:3231-3242.

Smith RD, Goldin AL (1998) Functional analysis of the rat I sodium channel in Xenopus oocytes. J Neurosci 18:811-820.

Taglialatela M, Toro L, Stefani E (1992) Novel voltage clamp to record small, fast currents from ion channels expressed in Xenopus oocytes. Biophys J 61:78-82.

Vega-Saenz de Miera E, Rudy B, Sugimori M, Llinas R (1997) Molecular characterization of the sodium channel subunits expressed in mammalian cerebellar Purkinje cells. Proc Natl Acad Sci USA 94: 7059-7064. 